\title{
Efficacy of communication-based interventions on physicians and patients' outcome: A meta-analysis of randomised controlled trials
}

\author{
Andreea Raluca ADAM, Florinda Tinela GOLU \\ Faculty of Psychology and Educational Sciences, University of Bucharest, Romania
}

\begin{abstract}
Communication-based intervention on physicians are strongly linked to patient health improvement. We proceeded examine the efficiency of these programs interventions for relevant outcomes in patients life, along with study quality, publication bias and potential moderators.

For this meta-analysis, we searched 2 databases, including: Web of Science and PubMed, using a comprehensive search strategy to identify the efficacy of the physician interventions on patient's outcome studies in the literature, published from inception of 1965. Studies reporting means of health-related outcome in patients were included in the analyses. Studies quality was assessed with risk of bias tool. Meta-regression was used to explore heterogeneity of the year of published studies moderator and subgroup analysis was used to explore the medical specialty moderator.

Of the 3,470 studies initially identified, 14 were eligible for inclusion. These studies had a large diversity of program interventions. The mean effect size representing the impact of communication intervention on patients physical and psychological health was not significant. The only significant mean effect was represented by psychological outcome. Significant associations were found between year of studies and general medicine specialization.

In conclusion the impact of physicians' intervention in not efficient on patients' communication and medical outcome but it improves psychological health.
\end{abstract}

Keywords: communication-based intervention, physician-patient relationship, patient

outcome, patient health

\section{INTRODUCTION}

The relationship between a doctor and patient is considered to be one of the most significant aspects of a good medical care. Within interactions in the mentioned dyad information is easily shared, choices more comfortable to be taken and reassurances are provided. In the medical relationship, trust is an essential emotional an behavioral element of effective treatment and is conceptually distinct from satisfaction with treatment (1) and physician-patient working alliance might be related to patients' beliefs about the usefulness of treatment. The patient satisfaction as an outcome of multiple factors, such number of patients a doctor sees within a day, accessibility to doctors, patients' use of alcohol and drugs, friendliness, and patient level of education (2) and it is also been linked with adherence to therapy, patients changing doctors, and outcome (3).

The increased interest in communication between physicians and patients is motivated by the aim to provide the best possible health care for patients and their 
families. In addition, studies indicate that effective and empathic communication skills may decrease the probability of patients filing malpractice claims against their physicians (4). Patients feeling rushed and ignored and physicians providing inadequate explanations and advice proved to be the most significant predictors of dissatisfaction and low outcomes in healthcare. The ability to communicate honestly and empathically with patients has a powerful effect on the success of a medical practice $(5,6)$.

\section{AIM}

The aim of the present meta-analysis is to examine whether there is robust empirical evidence for the efficacy of communication skills program interventions for physicians in the patients' health status.

\section{METHOD}

\section{Search strategy}

Studies were collected through searches of electronic databases Web of Science and PubMed, from the incept of 1965 to May 2020. A search strategy was developed for each database with a combination of free text and MeSH terms for controlling vocabulary. The following comprehensive search string was entered into the databases: "doctor psychological training" or "physician psychological training" or "doctor psychological intervention" or "physician psychological intervention" and "doctor-patient relationship" or "physician-patient relationship". Also, it was manually searching for citations in reference lists of the relevant articles and additional web-based search on Google Scholar for grey literature. The search was limited to those published in English language and human science, and each study from the final results was screened by title and abstract.

\section{Selection and eligibility criteria}

The selected studies were screened in two stages: screening of titles and abstracts followed by the retrieval and screening of full-text articles using the eligibility criteria recommended by the preferred reporting items for systematic reviews and meta-analyses (PRISMA) (7) in Figure 1.

Studies were included if they were applying the following conditions: the studies had a randomized controlled trial (RCT) design; studies that clearly delimited the population if interest from others (i.e. nurses or auxiliary personnel); an existing outcome from patients; patients evaluated the physicians (peer-report); an existing control group for both physicians and patients.

\section{Risk of bias}

The methodological quality of the included studies was assessed with risk of bias assessment tool, developed by the Cochrane Collaboration (8). The five criteria of sources of bias in RCTs assessment are: (a) adequate generation of allocation sequence; (b) concealment of allocation to conditions; (c) prevention of knowledge of the allocated intervention to assessors of outcome; (d) prevention of knowledge of the allocated intervention to participants and (e) dealing with incomplete data.

\section{Moderator variables}

We conducted categorical moderator analyses testing the one variable, the medical specialization of the physicians: general medicine, including specialties as: family medicine, surgery, military medicine; respectively oncology specialization, including any domain of the specialization. A second moderator, represent the year of studies publication, used as continuous moderator variable.

\section{Meta-analytic procedures}

We conducted a random effects meta-analysis with comprehensive meta-analysis software (CMA) version 3. The meta-analysis was conducted in the following sequence: First, we calculated the comparison between physician intervention group and a comparison group, the effect size, indicating the differences between the two groups at post-test. It was calculated using variance for Hedges'g. The effect size was calculated by means, standard deviation and number of participants (patients), at post-test with a control group. Outcome is the standardized mean difference for the patients from the trained physicians' group vs. the control group. Each study reports three outcomes at once or separately: medical, communication and/or psychological. Next, we conducted subgroup analyses for patient's outcome across all the studies included. In the subgroup analysis we consider each outcome as an independent study and a meta-regression analysis for publication year.

If means and standard deviations were not reported for the symptom outcomes in a study, we calculate the standardized mean difference from dichotomous data or from other statistics such as t-values or $\mathrm{p}$-values. If the effect size could possible to be calculated, the study was excluded. For the studies that had more than one intervention group or control group it was used the most relevant intervention group and only one control group, the less biased of intervention, to calculate effect size. From one study (9) was included with three point times outcomes (after intervention and follow up at 3 and 6 months). At the follow up the 
study has an additional outcome (medical outcome) for patients and different size of sample. For the rest of the studies we reported effect sizes for the time point available (e.g. post-test after intervention, 4 weeks later, 6 months or 12 months).

\section{RESULTS}

\section{Characteristics of the included studies}

Of the 3,470 studies, 2,510 potentially relevant fulltext articles were screened by title and abstract. From these, we identified 144 that that have had the potential to provide data on interventions on physicians and patients outcome were full text assessed. Among these, 26 examined intervention on physician in correlation with other variables, 14 provided communication intervention on nurses or patients, 26 had a different outcome (e.g. physician productivity or efficacy, physician wellbeing or mental health), 12 studies had lack outcome data, other than mean and standard de- viation (e.g. odd ratio or median) and could not be calculated by the author from the available data, in one study has not reported the patient outcome, five studies had the intervention on both physicians and nurses or other auxiliary personnel, two studies had no control group and other 4 had no intervention program. In 9 studies was no intervention and finally, 31 studies were qualitative studies, explaining a series of interventions by methodological and applying procedure. After excluding ineligible studies, 14 studies that provided the inclusion criteria data were included in the qualitative and quantitative processes as shows Figure 1.

\section{Risk of bias of the included studies}

Overall, the quality of the included RCTs was not optimal. Four studies met two high risk criteria out of the five considered. The highest risk of bias is targeting the random selection bias, followed allocation bias four studies out of 14 as seen in Figure 3. The percentage of
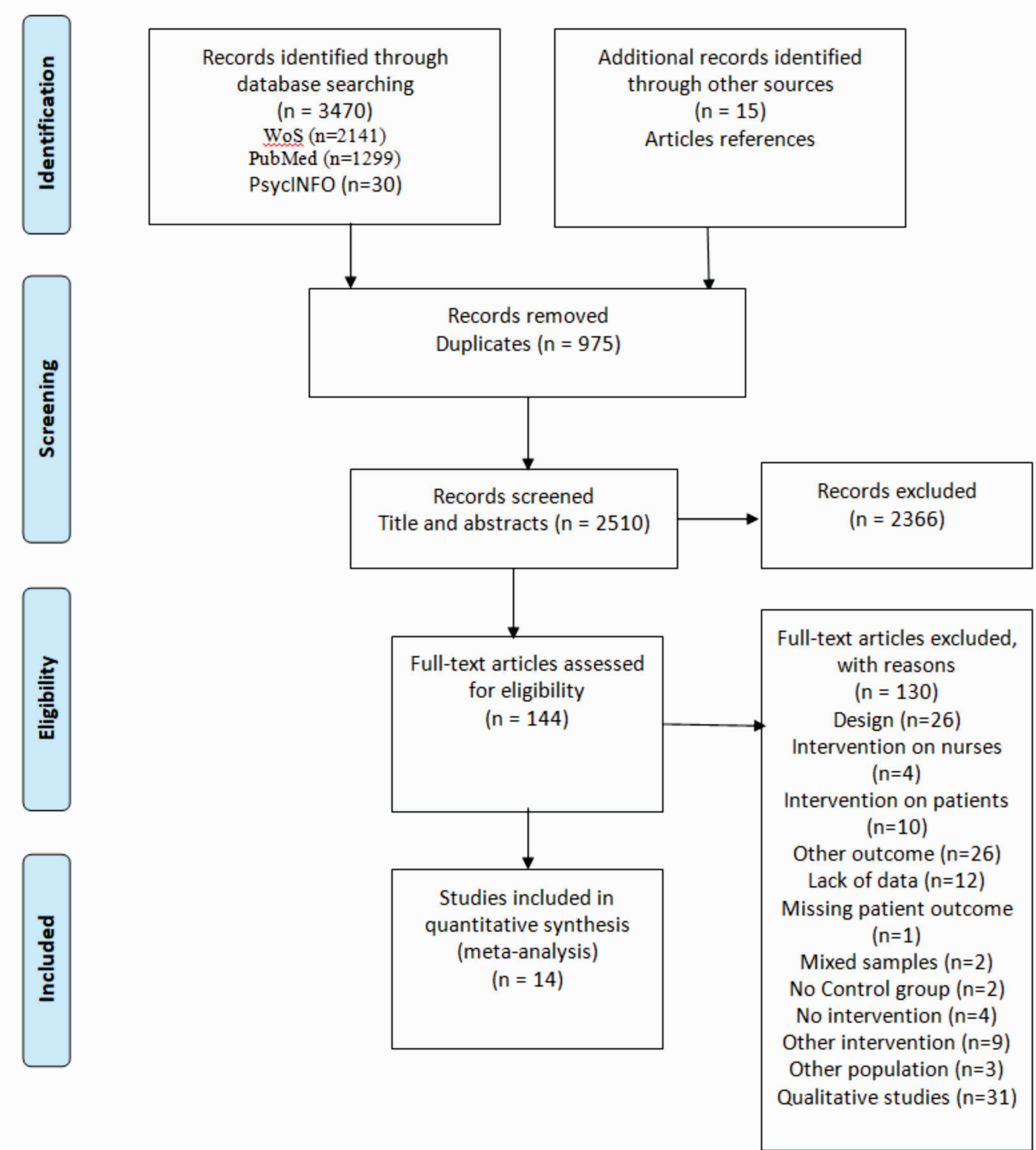

FIGURE 1. Flow chart of selection and inclusion process 


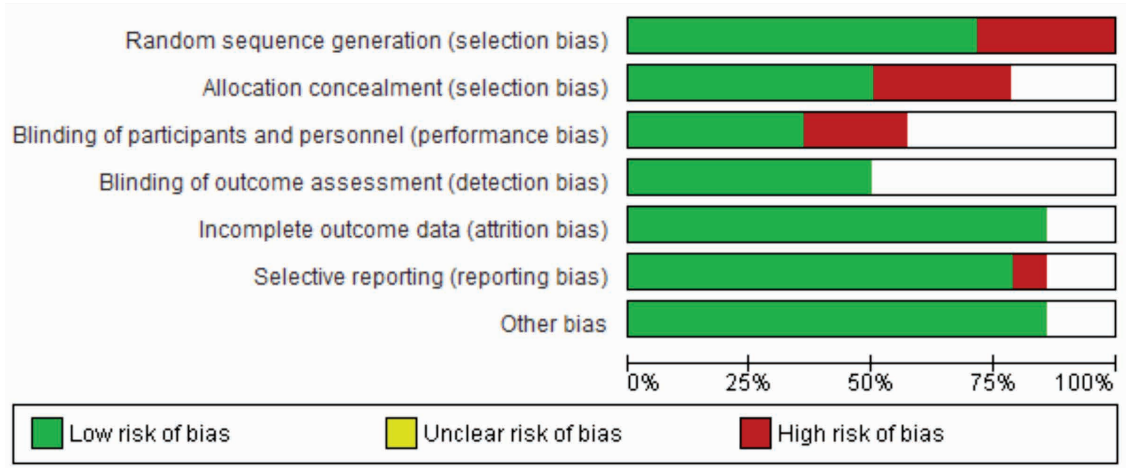

FIGURE 2. Risk of bias graph. Judgment about each risk of bias as per percentages

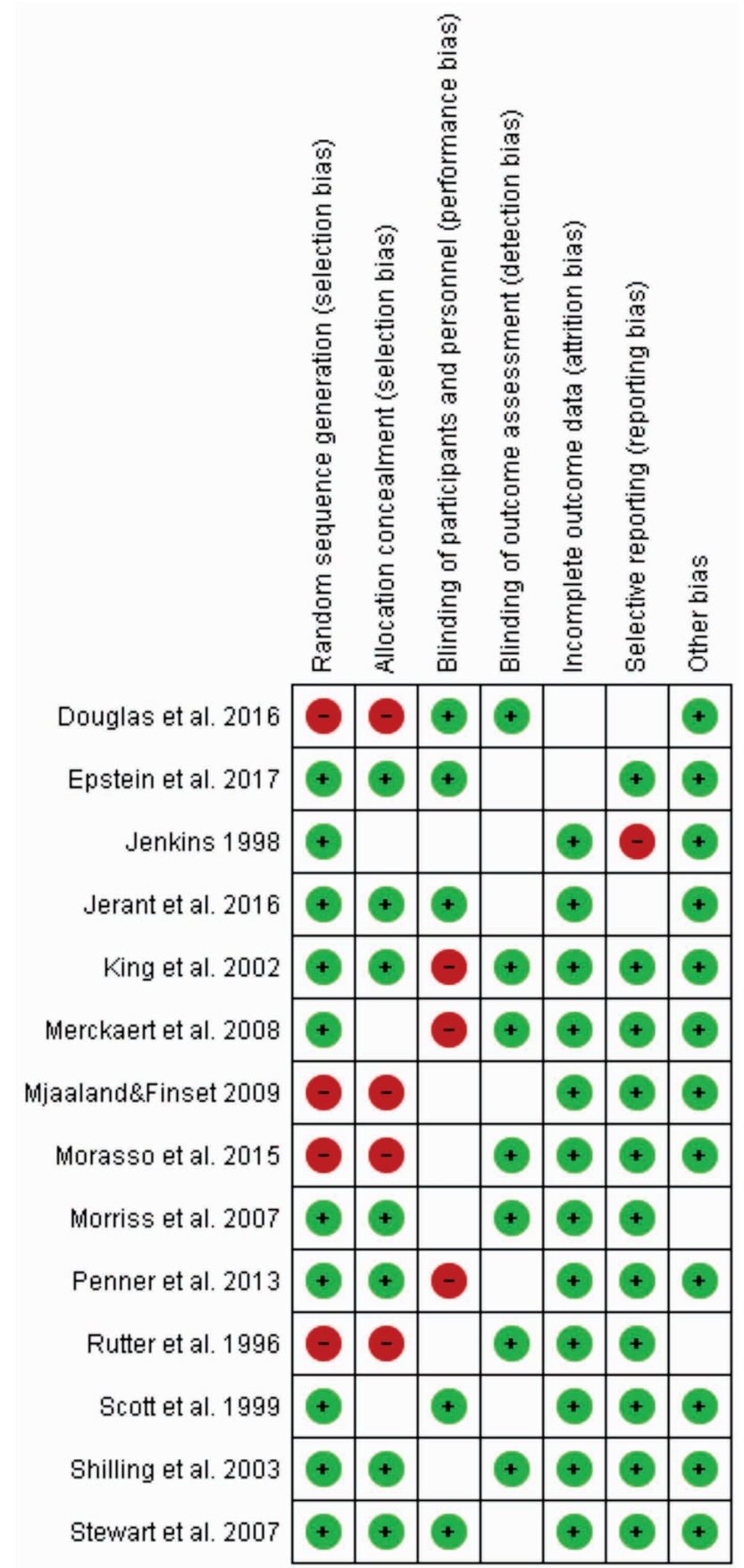

FIGURE 3. Sumarry of each study risk of bias each studies with a low, unclear and high risk of bias, for each of the quality criteria is presented in Figure 2. Low risk of bias was observed to outcome data criteria $(85 \%)$, the rest of $15 \%$ being considered as unclear.

Characteristics of the individual studies are reported in Table 1 . The $k=14$ studies, with program interventions based on physician communication and assessed with psychometric assessment tools.

MISS - Medical Interview Satisfaction Scale; PPSB Physician Psychosocial Belief; CAT - Communication Assessment Tool; PHDRA - Physician's Humanistic Behaviors Questionnaire; APPC - Active Patient Participation Coding; Verona VR-CoDES - Responding to patients' emotions; PTCC - Prognostic and Treatment Choices and Balanced Framing subscales; HBCM Perceived Medical Condition Self-Management Scale; BDI - Beck depression inventory; STAI - The State-Trait Anxiety Inventory; HADS - Hospital Anxiety and Depression Scale; PEQ - The Patient Experiences Questionnaire; PDI - Psychological Distress Inventory; STAI-S - State-Trait Anxiety Inventory-State; PSB - Patients' Symptom Beliefs; SF-20 - Self-perceived Racial and Ethnic Discrimination in Social Survey; PPDS Perceived Past Discrimination Scale; PSCQ - The Patient Satisfaction with Communication Questionnaire; CDIS - Cancer Diagnostic Interview Scale; BSY - Brief Symptom Inventory

\section{Efficacy of communication-based interventions}

The main meta-analytical results are displayed in Figure 4, the forest plot of the standardized effect sizes of communication interventions.

The mean effect size representing the impact of communication intervention on patients physical and psychological health is $\mathrm{g}=0.113(95 \% \mathrm{Cl}-0.070 ; 0.098)$ for all 14 RCTs, $(p>0.05)$. Heterogeneity was moderate $\left(I^{2}=32.11\right)$ (Table 2). The results show that the efficacy of the interventions is not significant. Next, we calculated the effect sizes for each outcome Figure 5 . The statistical analysis for communication outcome ( $N=6$, $\left.\mathrm{g}=0.169,95 \% \mathrm{Cl}=-0.160 ; 0.497, \mathrm{I}^{2}=21.79 ; \mathrm{p}>0.05\right)$, is not significant. The results point out that intervention 


\begin{tabular}{|c|c|c|c|c|c|c|c|c|c|c|c|c|c|c|c|c|}
\hline 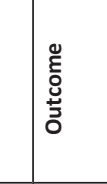 & 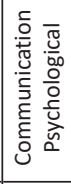 & 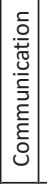 & 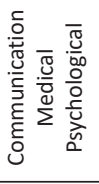 & 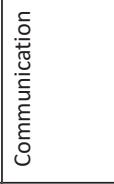 & 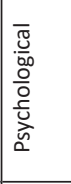 & 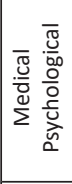 & 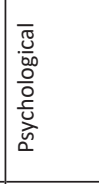 & 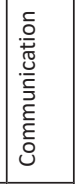 & 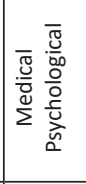 & 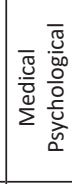 & 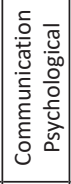 & 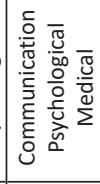 & 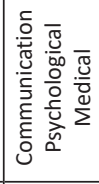 & 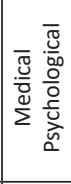 & 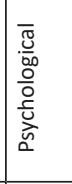 & 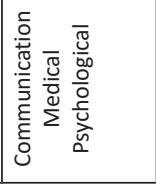 \\
\hline 章 & 9 & \& & $\stackrel{\infty}{\sim}$ & $\sigma$ & $\approx$ & ₹ & ని & 9 & न & $\infty$ & r & r & r & ' & 号 & $\stackrel{i}{\sim}$ \\
\hline 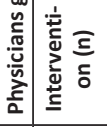 & 음 & $\stackrel{\infty}{q}$ & $\stackrel{\mathscr{N}}{\sim}$ & 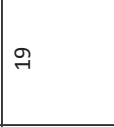 & $\grave{\Lambda}$ & F & $\grave{\curvearrowright}$ & $\stackrel{n}{\sim}$ & न & $\infty$ & r & - & r & m & $\stackrel{\check{g}}{\not}$ & $\stackrel{\sim}{N}$ \\
\hline 妾 & 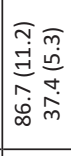 & 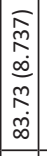 & 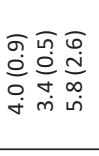 & \begin{tabular}{|l|}
0 \\
0 \\
0 \\
0 \\
0 \\
0 \\
0 \\
1 \\
1 \\
\end{tabular} & 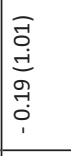 & 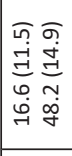 & 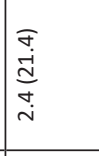 & $\begin{array}{l}\bar{\alpha} \\
\dot{\omega} \\
0 \\
\dot{b} \\
i n\end{array}$ & 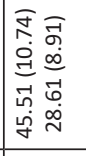 & 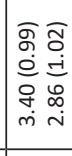 & 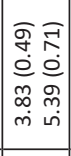 & 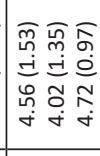 & 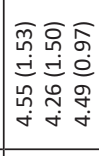 & 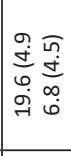 & 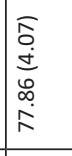 & 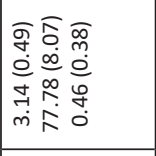 \\
\hline 妾 & $\begin{array}{l}\mathscr{0} \\
-1 \\
-1\end{array}$ & $\mid$ & ఏ్ & 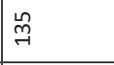 & ڤே & 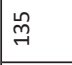 & ลิ & in & 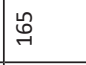 & $\aleph$ & \% & $\approx$ & A & $\stackrel{\infty}{-\infty}$ & $\tilde{\Im}$ & $\vec{\sigma}$ \\
\hline 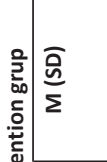 & 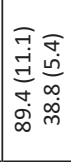 & 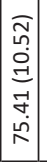 & 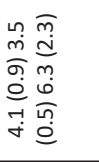 & \begin{tabular}{|l}
$\bar{\alpha}$ \\
$\dot{0}$ \\
$\dot{0}$ \\
0 \\
0 \\
0
\end{tabular} & $\begin{array}{l}\overline{0} \\
o \\
0 \\
0 \\
0 \\
0 \\
0\end{array}$ & 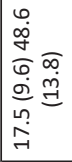 & $\begin{array}{l}\widehat{N} \\
\underset{N}{N} \\
\underset{N}{N} \\
\sim\end{array}$ & 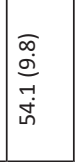 & 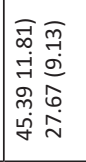 & 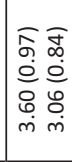 & 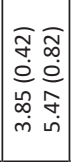 & 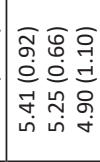 & 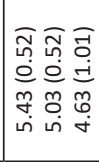 & 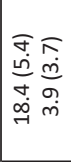 & 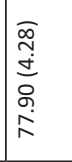 & 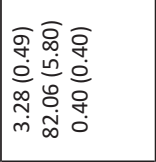 \\
\hline 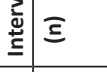 & 守 & \begin{tabular}{|l|l}
$\infty$ \\
$\stackrel{\sim}{\sim}$ \\
\end{tabular} & $\stackrel{9}{7}$ & 品 & $\mathscr{8}$ & 金 & i & $\widetilde{\sigma}$ & 亪 & $\bullet$ & z & i & i & $\stackrel{\infty}{\rightarrow}$ & 竎 & $\exists$ \\
\hline 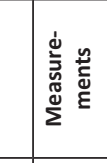 & $\frac{\tilde{n}}{\Sigma}$ & 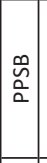 & 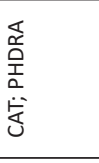 & 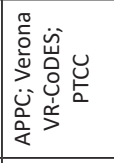 & $\mid \begin{array}{l}\sum_{0} \\
\text { 品 }\end{array}$ & $\mid$ & 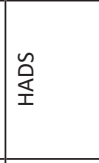 & 吕 & 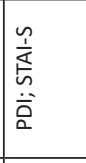 & 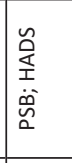 & 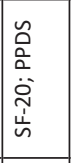 & 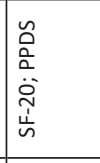 & 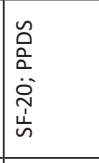 & $\mid \begin{array}{l}\text { 夏 } \\
\text { | }\end{array}$ & 怘 & 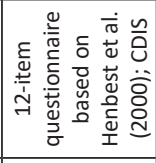 \\
\hline 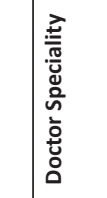 & 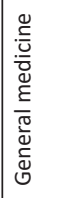 & 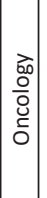 & 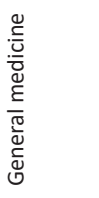 & 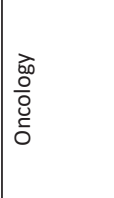 & 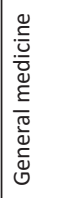 & 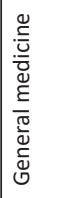 & 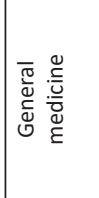 & 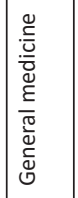 & 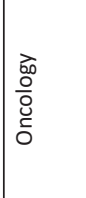 & 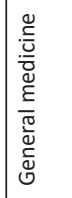 & 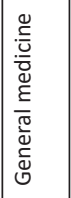 & 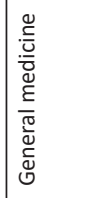 & 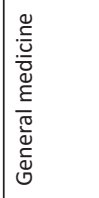 & 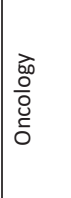 & 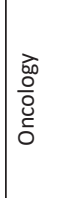 & 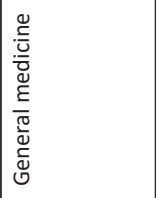 \\
\hline 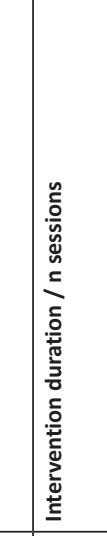 & 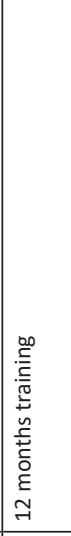 & 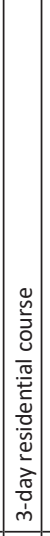 & 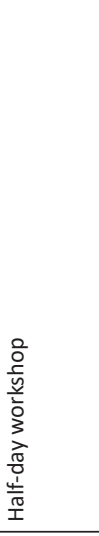 & 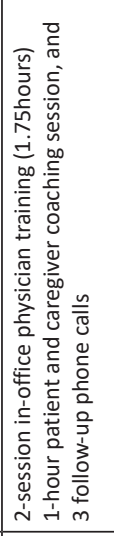 & 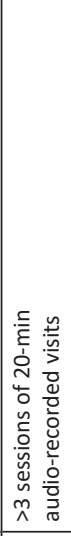 & 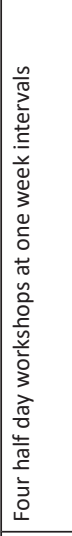 & 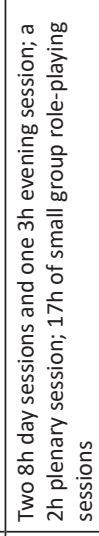 & 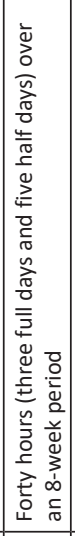 & 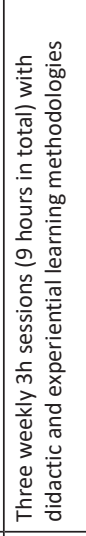 & 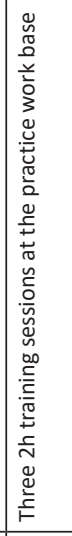 & & & & 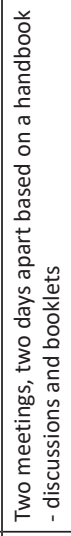 & 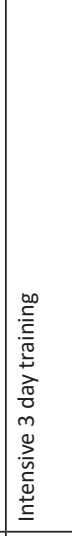 & 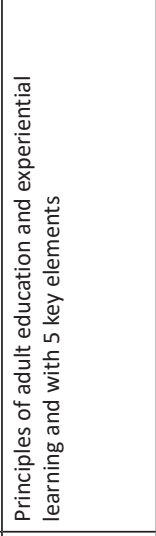 \\
\hline 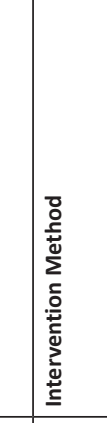 & 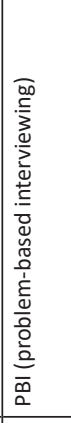 & 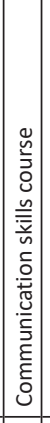 & 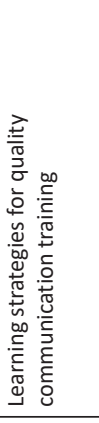 & 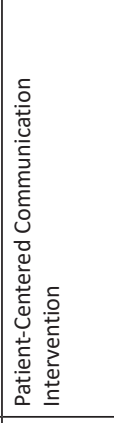 & 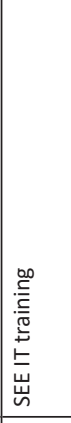 & 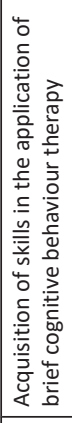 & 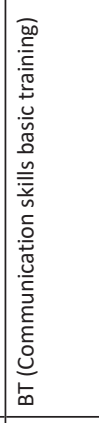 & 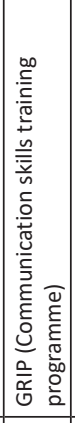 & 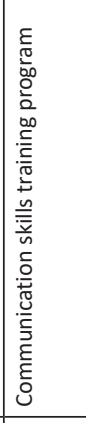 & 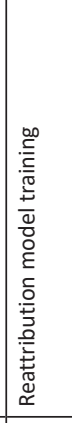 & 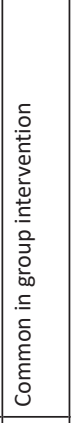 & 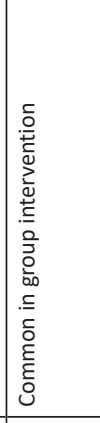 & 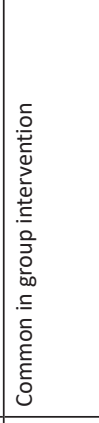 & 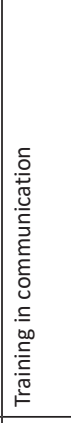 & 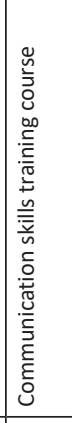 & 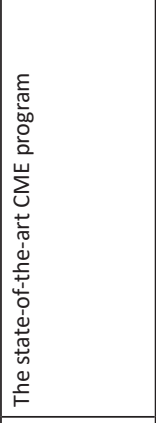 \\
\hline 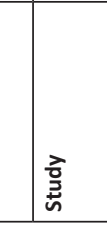 & 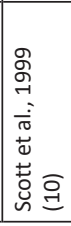 & 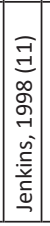 & 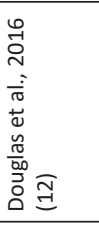 & 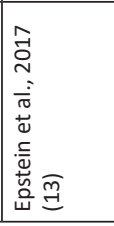 & 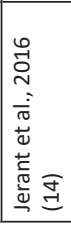 & 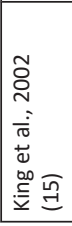 & 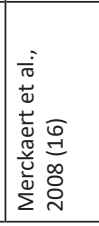 & 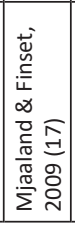 & 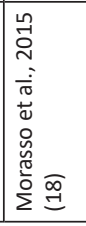 & 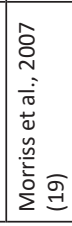 & 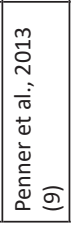 & 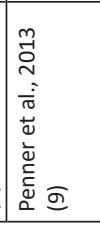 & 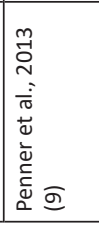 & 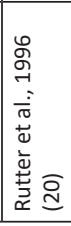 & 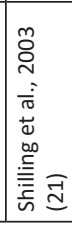 & 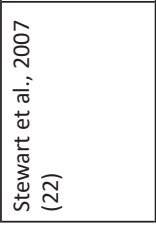 \\
\hline
\end{tabular}




\begin{tabular}{|c|c|c|c|c|c|c|c|c|}
\hline \multirow[t]{2}{*}{ Study name } & \multirow[t]{2}{*}{ Outcome } & \multicolumn{7}{|c|}{ Statistics for each study } \\
\hline & & $\begin{array}{l}\text { Hedges's } \\
\mathbf{g}\end{array}$ & $\begin{array}{l}\text { Standard } \\
\text { error }\end{array}$ & Variance & $\begin{array}{c}\text { Lower } \\
\text { limit }\end{array}$ & $\begin{array}{l}\text { Upper } \\
\text { limit }\end{array}$ & Z-Value & p-Value \\
\hline Scott et al., 1999 & Communication & 0.242 & 0.120 & 0.014 & 0.007 & 0.476 & 2.018 & 0.044 \\
\hline Jenkins, 1998 & Communication & -0.859 & 0.108 & 0.012 & -1.071 & -0.647 & -7.938 & 0.000 \\
\hline Douglas et al., 2016 & Communication & 0.111 & 0.053 & 0.003 & 0.006 & 0.216 & 2.076 & 0.038 \\
\hline Epstein et al., 2017 & Communication & 0.230 & 0.123 & 0.015 & -0.011 & 0.471 & 1.870 & 0.061 \\
\hline Jerant et al., 2016 & Psychological & 0.373 & 0.175 & 0.031 & 0.030 & 0.717 & 2.131 & 0.033 \\
\hline King et al., 2002 & Medical & 0.085 & 0.121 & 0.015 & -0.152 & 0.322 & 0.701 & 0.483 \\
\hline Merckaert et al., 2008 & Psychological & 0.442 & 0.267 & 0.071 & -0.081 & 0.965 & 1.655 & 0.098 \\
\hline Mjaaland \& Finset, 2009 & Communication & -0.241 & 0.181 & 0.033 & -0.596 & 0.115 & -1.328 & 0.184 \\
\hline Morasso et al., 2015 & Medical & -0.011 & 0.108 & 0.012 & -0.223 & 0.202 & -0.098 & 0.922 \\
\hline Morriss et al., 2007 & Medical & 0.203 & 0.168 & 0.028 & -0.127 & 0.533 & 1.205 & 0.228 \\
\hline Penner et al., 2013 & Communication & 0.102 & 0.237 & 0.056 & -0.362 & 0.566 & 0.431 & 0.667 \\
\hline Penner et al., 2013 (2) & Communication & 1.192 & 0.297 & 0.088 & 0.611 & 1.773 & 4.019 & 0.000 \\
\hline Penner et al., 2013 (3) & Communication & 0.666 & 0.315 & 0.099 & 0.048 & 1.284 & 2.114 & 0.035 \\
\hline Rutter et al., 1996 & Medical & -0.688 & 0.336 & 0.113 & -1.347 & -0.030 & -2.049 & 0.040 \\
\hline Shilling et al., 2003 & Psychological & 0.010 & 0.068 & 0.005 & -0.124 & 0.143 & 0.140 & 0.888 \\
\hline \multirow[t]{2}{*}{ Stewart et al., 2007} & Communication & 0.540 & 0.319 & 0.102 & -0.086 & 1.165 & 1.691 & 0.091 \\
\hline & & 0.113 & 0.093 & 0.009 & -0.070 & 0.296 & 1.205 & 0.228 \\
\hline
\end{tabular}

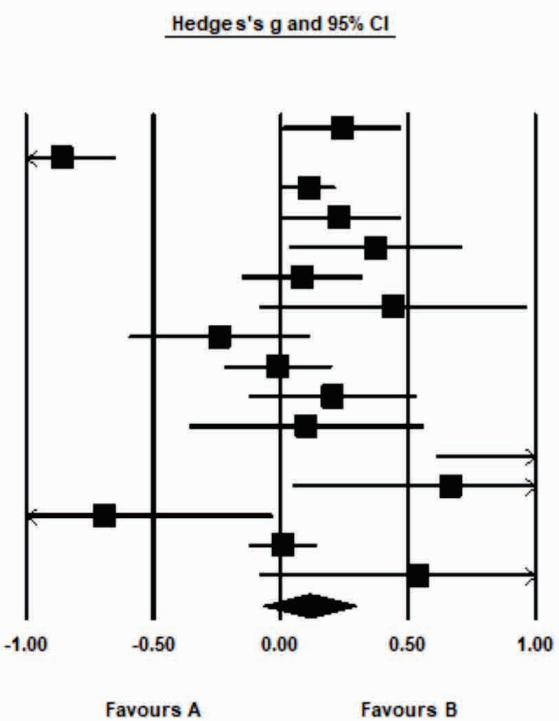

FIGURE 4. Statistics for studies included

\begin{tabular}{|c|c|c|c|c|c|c|c|c|c|}
\hline \multirow{2}{*}{$\begin{array}{l}\text { Group by } \\
\text { Outcome }\end{array}$} & \multirow[t]{2}{*}{ Study name } & \multirow[t]{2}{*}{ Outcome } & \multicolumn{7}{|c|}{ Statistics for each study } \\
\hline & & & $\begin{array}{c}\text { Hedges's } \\
g\end{array}$ & $\begin{array}{l}\text { Standard } \\
\text { error }\end{array}$ & Variance & $\begin{array}{l}\text { Lower } \\
\text { limit }\end{array}$ & $\begin{array}{l}\text { Upper } \\
\text { limit }\end{array}$ & Z-Value & p-Value \\
\hline Conmuniction & Soott et al. 1999 & Conmunicstion & 0.242 & 0.120 & 0.014 & 0.007 & 0.478 & 2018 & 0.044 \\
\hline Conmenication & Jentirs, 1988 & Commenication & -0.869 & 0.108 & 0.012 & -1.071 & -0.847 & -7.968 & 0.000 \\
\hline Conmunication & Dougles e $\mathrm{A}$, , 2018 & Commenication & 0.111 & 0.058 & 0003 & 0.008 & 0216 & $20 \pi 8$ & 0.038 \\
\hline Cormunication & Essteinetal, 2017 & Commenication & 0230 & 0.123 & 0.015 & -0.011 & 0.471 & 1.870 & 0.081 \\
\hline Commeniction & Mjasalard \& Finset 2000 & Commeniction & -0.241 & 0.181 & 0033 & -0.508 & 0.115 & -1.328 & 0.184 \\
\hline Cormunication & Penne etsl, 2013 & Communicaion & 0.102 & 0.23 & 0.050 & -0322 & 0.500 & 0.41 & 0.007 \\
\hline Conmunication & Penng etal, $2013(2)$ & Conmunicsion & 1,192 & 0.297 & 0088 & 0.011 & $1.7 \pi 3$ & 4019 & 0.000 \\
\hline Communication & Fenne etal., 2013 (3) & Comminicsion & 0008 & 0.315 & 0.099 & 0.048 & 1284 & 2114 & 0.005 \\
\hline Communiation & Stevertetal, 2007 & Commenication & 0.540 & 0.319 & 0,02 & -0.006 & 1.186 & 1.001 & 0.091 \\
\hline Commenication & & & a.e् & 0.188 & 0.028 & -0.160 & a.497 & 1.008 & 0.315 \\
\hline Mecical & Douplas \& $\mathrm{A}$., 2018 & Medical & 0204 & 0.054 & 0003 & 0.099 & 0309 & 3008 & 0.000 \\
\hline Mecical & King $\$$ al., adCe & Medical & 0085 & 0.121 & 0015 & -0.152 & 0.322 & 0.701 & 0.83 \\
\hline Mecical & Morass o e al, 2015 & Medical & .0 .011 & 0.108 & 0.012 & -0.223 & 0.202 & $-0.0 \%$ & 0.522 \\
\hline Medical & Morriss et al. 2007 & Medical & 0203 & 0.168 & 0028 & -0.127 & 0.533 & 1.205 & 0.228 \\
\hline Medical & Penng et al, 2013 (2) & Medical & 0.170 & 0.274 & 0075 & -.0387 & 0708 & 0,020 & 0.505 \\
\hline Mecical & Perng etal, 2013 (3) & Medical & 0.139 & 0.307 & 0094 & .0 .03 & 0.740 & 0.62 & 0.651 \\
\hline Mecical & Rutter \& d d., 1998 & Medical & .0088 & 0.336 & $a_{113}$ & -1.347 & -0.030 & .2000 & 0.040 \\
\hline Mecical & Stencr tetal, 2007 & Medical & .0150 & 0.317 & 0.100 & $-a \pi \pi$ & 0.465 & -0.492 & 0.023 \\
\hline Meciaral & & & 0.088 & 0.067 & 0.004 & -0.043 & 0.219 & 1.315 & 0.189 \\
\hline Psychological & Scott et 11.1999 & Psychological & 0.261 & 0.120 & 0.014 & 0.028 & 0.498 & 2179 & 0.029 \\
\hline Paychological & 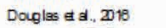 & Psychological & 0200 & 0.054 & 0.003 & 0.096 & 0.305 & 3731 & 0.000 \\
\hline Psychological & Jast \& $d .2018$ & Psychological & 0.373 & 0.175 & 0.031 & 0.030 & 0.717 & 2131 & 0.003 \\
\hline Psyctological & King a $\mathrm{al}$. $20 \subset$ & Psychological & 0.028 & 0.121 & 0.015 & -0.209 & 0285 & 0.230 & 0.818 \\
\hline Psychological & Merdea tet $8 ., 2008$ & Psychological & 0.442 & 0.267 & 0.071 & -0.081 & 0995 & 1.665 & 0.088 \\
\hline Psychological & Morass o e al., 2015 & P3ychological & -0.104 & 0.108 & 0.012 & -0.317 & 0.109 & -0.988 & 0.338 \\
\hline Psyctological & Morriss etal, 2007 & Psychological & 0212 & 0.188 & 0.028 & -0.118 & 0.542 & 1.257 & 0.209 \\
\hline Psycrological & Fenne etal., 2013 & Psychological & 0.044 & 0237 & 0050 & -0.420 & 0.507 & 0,186 & 0.853 \\
\hline Psycrological & Fenne stal., $2013(2)$ & Psychologioal & oese & 0.281 & 0.079 & 0.135 & 1237 & 2440 & 0.016 \\
\hline Psycrological & Fenng etsi, 2013 (3) & Psychological & 0.748 & 0.317 & a.101 & 0.126 & 1370 & 2360 & 0.018 \\
\hline Psycrological & Ruteres at d. 1998 & Psychological & -0.228 & 0.327 & Q.107 & -0.829 & 0.413 & -0.098 & 0.87 \\
\hline Psycrological & Shilligetal. 2003 & Psychological & 0010 & 0.088 & 0.005 & -0.124 & 0.143 & 0.140 & 0.288 \\
\hline Psycrological & Stencritet 1., 2007 & Psychological & 0284 & 0.317 & a.101 & -0.339 & 0.908 & 0.898 & 0.32 \\
\hline Psyctological & & & 0.181 & 0.058 & 0.003 & 0.048 & 0.274 & 2801 & 0.005 \\
\hline
\end{tabular}

Pasctological

$\begin{array}{lllll}0.003 & 0.048 & 0.274 & 2901 & 0.005\end{array}$

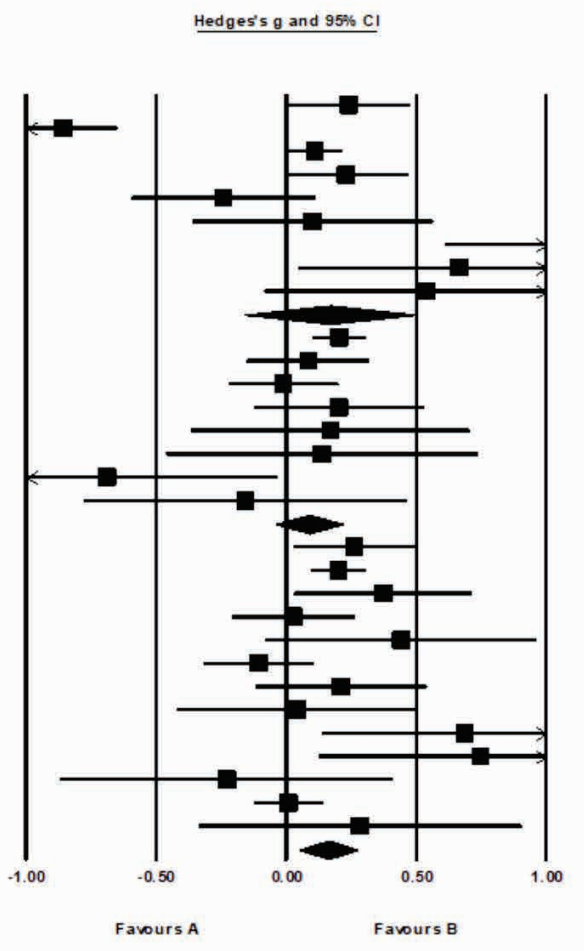

FIGURE 5. Statistics for each outcome

programs do not improve the communication between physician and patient. The effect size for medical outcome $(\mathrm{N}=8, \mathrm{~g}=0.088,95 \% \mathrm{Cl}=-0.043 ; 0.219$, $\left.I^{2}=4.134 ; p>0.05\right)$ is not significant. Finally, for psychological outcome $(\mathrm{N}=13, \mathrm{~g}=0.161,95 \% \mathrm{Cl}=0.003$; $\left.0.048, I^{2}=12.75 ; p<0.05\right)$ is significant, therefore intervention programs applied on physicians support a high- er level of psychological state improvement in patients. Figure 6 represents the funnel plots as follows: (a) mixed outcomes; (b) communication outcome; (c) medical outcome and (d) psychological outcome. These effects were not symmetrical, and the trim and fill analysis suggested adjustment to these mean effect sizes (23). 
TABLE 2. Standardized effect sizing of interventions and of medical specialty moderator

\begin{tabular}{|l|l|c|c|c|c|c|c|c|c|}
\hline Model & Outcome & $\mathbf{N}-\mathbf{c o m p}$ & $\mathbf{g}(\mathbf{9 5 \%} \mathbf{~ C l})$ & Std. er. & Variance & LL & UL & $\mathbf{Z}$ & $\mathbf{I}^{\mathbf{2}}$ \\
\hline Fixed & Mixed & 14 & 0.040 & 0.030 & 0.001 & -0.019 & 0.098 & 1.318 & 86.612 \\
\hline Random & Mixed & 14 & 0.113 & 0.093 & 0.009 & -0.070 & 0.296 & 1.205 & 32.110 \\
\hline Fixed & Communication & 6 & 0.027 & 0.039 & 0.002 & -0.050 & 0.104 & 0.491 & 91.988 \\
\hline Random & Communication & 6 & 0.169 & 0.168 & 0.160 & -0.160 & 0.497 & 0.315 & 21.787 \\
\hline Fixed & Medical & 8 & 0.137 & 0.042 & 0.002 & 0.055 & 0.218 & $3.288^{*}$ & 18.756 \\
\hline Random & Medical & 8 & 0.088 & 0.067 & 0.004 & -0.043 & 0.219 & 1.315 & 4.134 \\
\hline Fixed & Psychological & 13 & 0.136 & 0.033 & 0.001 & 0.072 & 0.200 & $4.147^{*}$ & 50.293 \\
\hline Random & Psychological & 13 & 0.161 & 0.058 & 0.003 & 0.048 & 0.274 & $2.801^{*}$ & 12.746 \\
\hline Fixed & General Medicine & 10 & 0.159 & 0.040 & 0.002 & 0.082 & 0.237 & $4.024^{*}$ & 62.464 \\
\hline Random & General Medicine & 10 & 0.234 & 0.082 & 0.007 & 0.073 & 0.395 & $2.855^{*}$ & 35.340 \\
\hline Fixed & Oncology & 6 & -0.123 & 0.046 & 0.002 & 0.032 & -2.664 & $0.008^{*}$ & 92.482 \\
\hline Random & Oncology & 6 & -0.134 & 0.192 & 0.037 & -0.509 & 0.242 & -0.698 & 18.308 \\
\hline
\end{tabular}

${ }^{*} p<0.05 ; \mathrm{N}$-comp - number of participants; Std. er. - standard error; LL - lower limit; UL - Upper limit; $I^{2}$ - heterogenity

\section{Intervention moderators}

The subgroup analysis of medical specialty revealed no statistically significant differences among oncology specialty $(\mathrm{N}=6, \mathrm{~g}=-0.134,95 \% \mathrm{Cl}=-0.509 ; 0.242$, $\left.\mathrm{I}^{2}=18.308 ; \mathrm{p}>0.05\right)$ but significant result in general medicine specialty $(\mathrm{N}=10, \mathrm{~g}=0.234,95 \% \mathrm{Cl}=0.073$; $\left.0.395, \mathrm{I}^{2}=35.34 ; \mathrm{p}<0.05\right)$ and medium heterogeneity (Table 2). Therefore, the impact of the communication interventions on physician is higher for oncology patients.

The range of the years studied were published, vary between 1996 and 2017. The results of meta-regression analysis are displayed in Table 3 . Statistical analyses are positive assuming that there is a significant impact of year of publication on effect sizes $(Z=2.48$, $95 \% \mathrm{Cl}=0.0069 ; 0.059, \mathrm{p}<0.000)$ and the heterogeneity was high $\left(I^{2}=86.61\right)$, indicating that more recent studies reported higher effects.

\section{DISCUSSIONS}

The purpose of this study was to examine the efficacy of communication programs interventions on physicians to improve health behaviors and health-related outcomes in patients. We conducted a meta-analysis including all communication interventions on physicians tested in RCTs for relevant outcomes on patients. Interventions were considered as therapeutic programs to facilitate a better relationship between physician and patient and improvement in patients psychological and medical condition. We examined the intervention effects for clinical patients, subclinical and nonclinical participants. We also analyzed two general moderators, one from the perspective of physician characteristics and one from the studies characteristics, with possible implication for further studies.

From all 3,470 studies 14 were selected. Overall, the meta-analysis included 1,390 physicians, 710 in the intervention group and 680 in the control group. From all 4,583 patients who rate the physician's changes, 2,271 were in the intervention group and the rest of 2,312 patients in the control group. Patients measured outcomes were diverse and clustered in three main categories: (a) communication outcome (satisfaction with the medical interview, patient centeredness, doctor-centeredness, inter-personal skills, physician trust or satisfaction with the consultation); (b) medical outcome (treatment adherence, clinical anxiety and depression, physical health concerns or shorter duration of symptoms) and (c) psychological outcome (sub-clinical anxiety, affection, mental health concerns, self-management, psychological distress or psychological impact on life).

Our first research question asked whether better communication skills in physicians can improve the relationship between physician-patient and moreover if there are any health outcomes on patient. Results revealed that these interventions are not generally efficacious $(24,25)$. Interventions applied on physicians attempted to improve only the psychological outcomes. On psychological level, a safe physician-patient relationship involves patient participation in decision-making and enhance in patient a higher level of self-determination (26). For the second question, whether medical specialty and year of the studies are significant moderation, results reviled that recent communication-based intervention are more efficient. General practitioners show more communication skills and attention to patient concerns and create an affective atmosphere for patient wellbeing (27).

This meta-analysis has a series of limitations. First, the small number of RCTs studies limited the analysis to 14 included studies. For further research it is recommended to be included RCTs with more than one follow-up time and longitudinal studies. The intervention programs vary from a study to another. Each program had its own methodological and duration of the inter- 


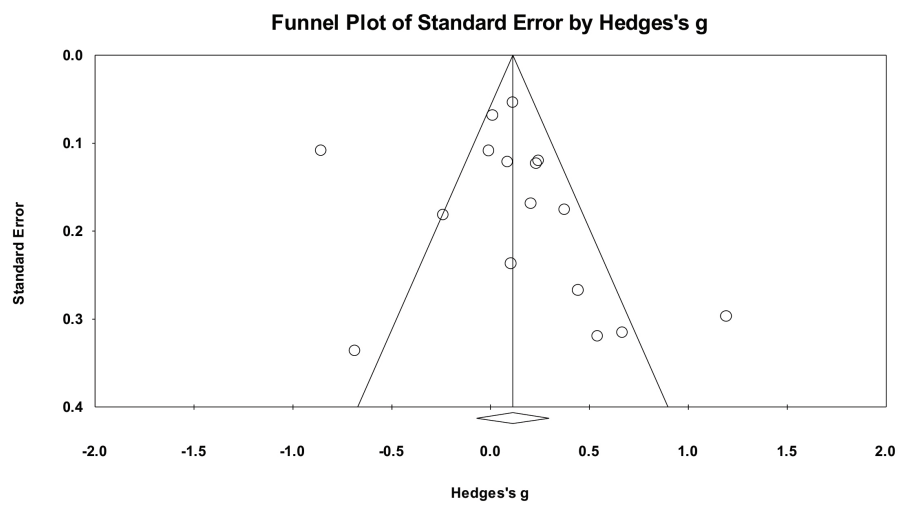

Funnel Plot of Standard Error by Hedges's $\mathbf{g}$

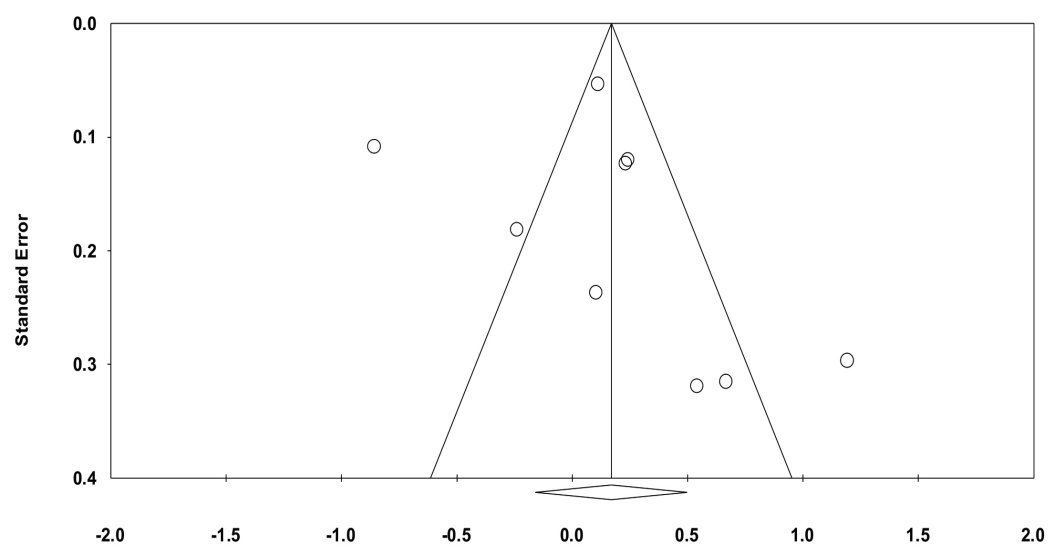

Funnel Plot of Standard Error by Hedges's $\mathbf{g}$

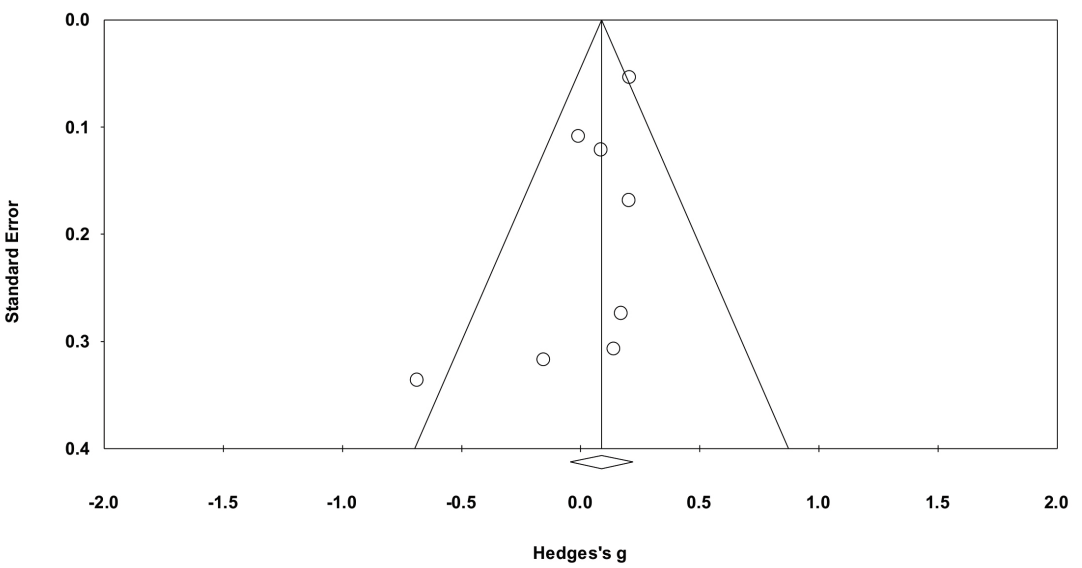

Funnel Plot of Standard Error by Hedges's g

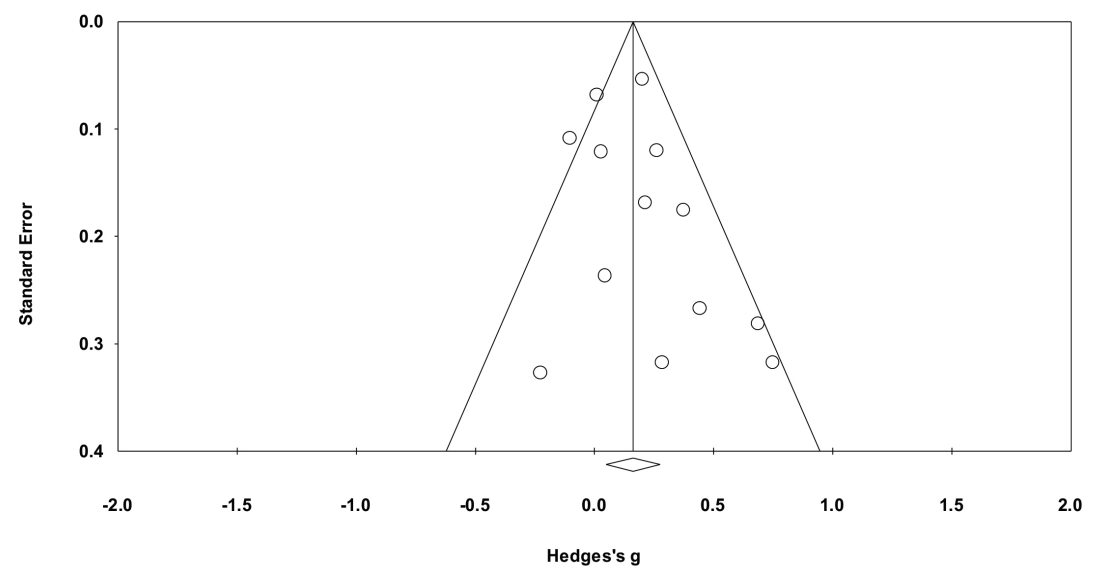

FIGURE 6. Funnel plots of standard error for outcomes 
TABLE 3. Statistics for the meta-regression model

\begin{tabular}{|l|c|c|c|c|c|c|c|c|c|}
\hline & Coefficient & Std. error & LL & UL & Z-value & Tau $^{2}$ & $\mathbf{I}^{\mathbf{2}}$ & $\mathbf{Q}$ & $\mathbf{p}$ \\
\hline Publication Year & 0.033 & 0.0133 & 0.0069 & 0.059 & $2.48^{*}$ & 0.0966 & 86.61 & 87.25 & 0.000 \\
\hline
\end{tabular}

${ }^{*} p<0.05$; Std. error-standard error; $L L$ - lower limit; UL - upper limit; $I^{2}$-heterogenity

\section{Regression of Hedges's g on Publication Year}

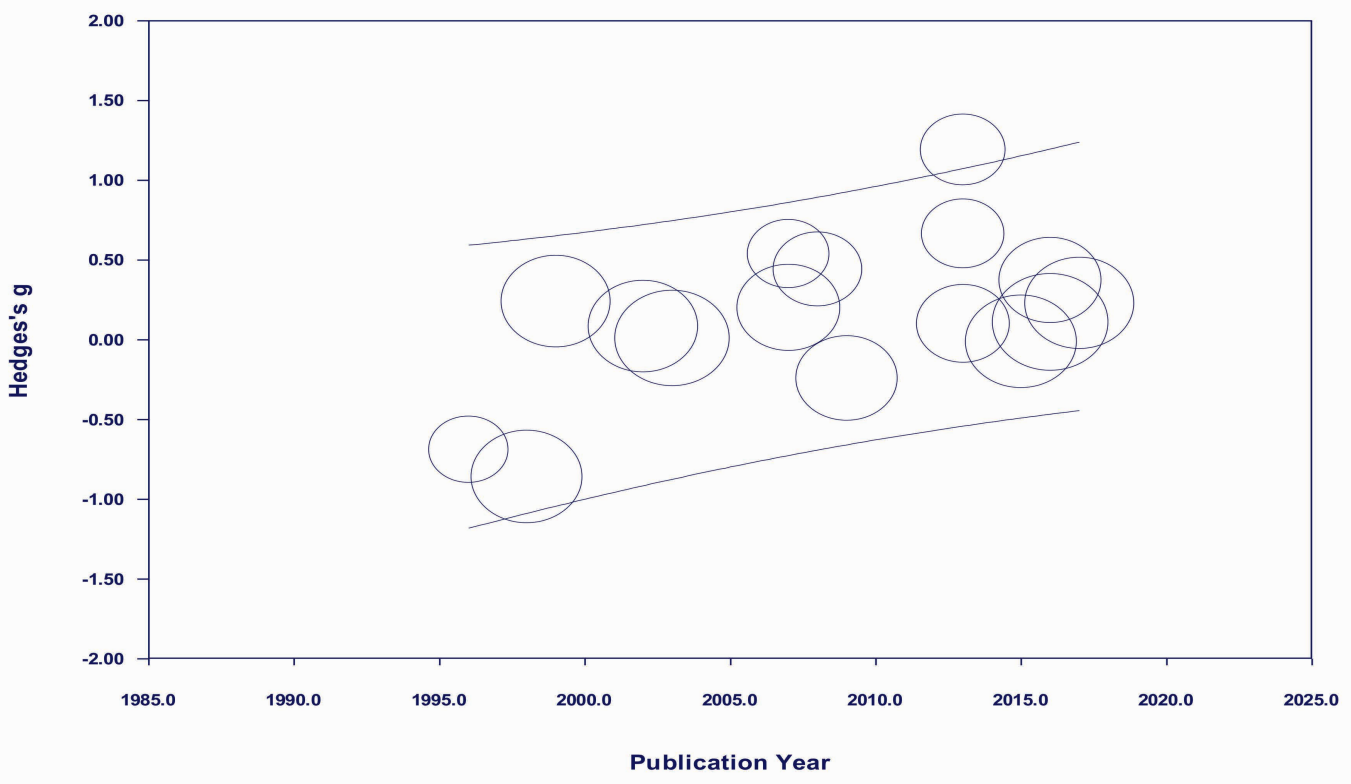

FIGURE 7. Regression plot of publication year

vention that can contribute to different outcomes in patients' condition. Some potentially important moderators such as severity of patients' symptoms and socioeconomic variables (e.g., ethnicity, education) could be considered. Future research should therefore consider the impact of these important moderators.

\section{CONCLUSIONS}

The results points it is necessary to set a goal development of an universal training in communication skills for physicians and the application of the new communication skills in daily practice to improve the patient's health and satisfaction with the medical care and treatment. Our results somewhat support the assumption that the transfer of communication skills into a real consultation with the patient is hard to apply and it is important to start to research more in this domain.

\section{REFERENCES}

1. Thom DH, Hall MA, Pawlson LG. Measuring patients' trust in physicians when assessing quality of care. Health Aff (Millwood). 2004;23(4):124-32.

2. Concato J, Feinstein AR. Asking patients what they like: overlooked attributes of patient satisfaction with primary care. The American Journal of Medicine 1997;102(4):399-406.

3. Kaplan SH, Greenfield S, Ware JE Jr. Assessing the effects of physician-patient interactions on the outcomes of chronic disease. Med Care. 1989 Mar;27(3 Suppl):S110-27.

4. Duffy FD, Gordon GH, Whelan G, Cole-Kelly $\mathrm{K}$, et al.; Participants in the American Academy on Physician and Patient's Conference on Education and Evaluation of Competence in Communication and Interpersonal Skills. Assessing competence in communication and interpersonal skills: the Kalamazoo II report. Acad Med. 2004 Jun;79(6):495-507.
Conflict of interest: none declared Financial support: none declared
5. Brenner RJ, Bartholomew L. Communication errors in radiology: a liability cost analysis. J Am Coll Radiol. 2005 May;2(5):428-31.

6. Forster HP, Schwartz J, DeRenzo E. Reducing legal risk by practicing patientcentered medicine. Arch Intern Med. 2002 Jun 10;162(11):1217-9.

7. Moher D, Liberati A, Tetzlaff J, Altman DG; PRISMA Group. Preferred reporting items for systematic reviews and meta-analyses: the PRISMA statement. Ann Intern Med. 2009 Aug 18;151(4):264-9, W64. 
8. Higgins JP, Altman DG, Gøtzsche PC, Jüni P, Moher D, et al.; Cochrane Bias Methods Group; Cochrane Statistical Methods Group. The Cochrane Collaboration's tool for assessing risk of bias in randomised trials. BMJ. 2011 Oct 18;343:d5928.

9. Penner LA, Gaertner S, Dovidio JF, Hagiwara N, et al. A social psychological approach to improving the outcomes of racially discordant medical interactions. J Gen Intern Med. 2013 Sep;28(9):1143-9.

10. Scott J, Jennings T, Standart S, Ward R, Goldberg $D$. The impact of training in problem-based interviewing on the detection and management of psychological problems presenting in primary care. Br J Gen Pract. 1999 Jun;49(443):441-5.

11. Jenkins SR. Emergency medical workers' mass shooting incident stress and psychological recovery. International Journal of Mass Emergencies and Disasters 1998;16(2):181-197.

12. Douglas SR, Vides de Andrade AR, Boyd S, Leslie M, et al. Communication training improves patient-centered provider behavior and screening for soldiers' mental health concerns. Patient Educ Couns. 2016 Jul;99(7):1203-1212.

13. Epstein RM, Duberstein PR, Fenton JJ, Fiscella K, et al. Effect of a Patient-Centered Communication Intervention on OncologistPatient Communication, Quality of Life, and Health Care Utilization in Advanced Cancer: The VOICE Randomized Clinical Trial. JAMA Oncol. 2017 Jan 1;3(1):92-100.

14. Jerant A, Lichte M, Kravitz RL, Tancredi DJ, Magnan EM, Hudnut A, Franks P. Physician training in self-efficacy enhancing interviewing techniques (SEE IT): Effects on patient psychological health behavior change mediators. Patient Educ Couns. 2016 Nov;99(11):1865-1872.

15. King M, Davidson O, Taylor F, Haines A, Sharp D, Turner R. Effectiveness of teaching general practitioners skills in brief cognitive behaviour therapy to treat patients with depression: randomised controlled trial. BMJ. 2002 Apr 20;324(7343):947-50.

16. Merckaert I, Libert Y, Delvaux N, Marchal S, Boniver J, et al. Factors influencing physicians' detection of cancer patients' and relatives' distress: can a communication skills training program improve physicians' detection? Psychooncology. 2008 Mar;17(3):260-9.

17. Mjaaland TA, Finset A. Communication skills training for general practitioners to promote patient coping: the GRIP approach. Patient Educ Couns. 2009 Jul;76(1):84-90.

18. Morasso G, Caruso A, Belbusti V, Carucci T, Chiorri $\mathrm{C}$, et al. Improving physicians' communication skills and reducing cancer patients' anxiety: a quasi-experimental study. Tumori. 2015 Jan-Feb;101(1):131-7.

19. Morriss R, Dowrick C, Salmon P, Peters S, Dunn $G$, et al. Cluster randomised controlled trial of training practices in reattribution for medically unexplained symptoms. $\mathrm{Br} \mathrm{J}$ Psychiatry. 2007 Dec;191:536-42.

20. Rutter DR, Iconomou G, Quine L. Doctorpatient communication and outcome in cancer patients: An intervention. Psychology and Health 1996;12(1):57-71.
21. Shilling V, Jenkins V, Fallowfield L. Factors affecting patient and clinician satisfaction with the clinical consultation: can communication skills training for clinicians improve satisfaction? Psychooncology. 2003 Sep;12(6):599-611.

22. Stewart M, Brown JB, Hammerton J, Donner A, Gavin A, et al. Improving communication between doctors and breast cancer patients. Ann Fam Med. 2007 Sep-Oct;5(5):387-94.

23. Duval S, Tweedie R. Trim and fill: A simple funnel-plot-based method of testing and adjusting for publication bias in metaanalysis. Biometrics. 2000 Jun;56(2):455-63.

24. Henrikson NB, Opel DJ, Grothaus L, Nelson J, Scrol A, et al. Physician Communication Training and Parental Vaccine Hesitancy: A Randomized Trial. Pediatrics. 2015 Jul;136(1):70-9.

25. Joos SK, Hickam DH, Gordon GH, Baker LH. Effects of a physician communication intervention on patient care outcomes. J Gen Intern Med. 1996 Mar;11(3):147-55.

26. Guadagnoli E, Ward P. Patient participation in decision-making. Soc Sci Med. 1998 Aug;47(3):329-39.

27. de Ridder DT, Theunissen NC, van Dulmen $\mathrm{SM}$. Does training general practitioners to elicit patients' illness representations and action plans influence their communication as a whole? Patient Educ Couns. 2007 Jun;66(3):327-36. 